\title{
Effects of emotional arousal in the cerebral hemispheres: a study of oscillatory brain activity and event-related potentials
}

\author{
Andreas Keil ${ }^{\mathrm{a}, *}$, Matthias M. Müller ${ }^{\mathrm{b}}$, Thomas Gruber ${ }^{\mathrm{b}}$, Christian Wienbruch ${ }^{\mathrm{a}}$, \\ Margarita Stolarova $^{\mathrm{a}, \mathrm{c}}$, Thomas Elbert ${ }^{\mathrm{a}}$ \\ ${ }^{a}$ Department of Psychology, University of Konstanz, D-78434 Konstanz, Germany \\ ${ }^{\mathrm{b}}$ Cognitive Neuroscience, Department of Psychology, University of Liverpool, Liverpool, UK \\ ${ }^{\mathrm{c}}$ Institute of Child Development, University of Minnesota, Minneapolis, MN, USA
}

Accepted 2 August 2001

\begin{abstract}
Objective: The present study aimed at examining the time course and topography of oscillatory brain activity and event-related potentials (ERPs) in response to laterally presented affective pictures.

Methods: Electroencephalography was recorded from 129 electrodes in 10 healthy university students during presentation of pictures from the international affective picture system. Frequency measures and ERPs were obtained for pleasant, neutral, and unpleasant pictures.

Results: In accordance with previous reports, a modulation of the late positive ERP wave at parietal recording sites was found as a function of emotional arousal. Early mid gamma band activity (GBA; 30-45 Hz) at $80 \mathrm{~ms}$ post-stimulus was enhanced in response to aversive stimuli only, whereas the higher GBA (46-65 Hz) at $500 \mathrm{~ms}$ showed an enhancement of arousing, compared to neutral pictures. ERP and late gamma effects showed a pronounced right-hemisphere preponderance, but differed in terms of topographical distribution.

Conclusions: Late gamma activity may represent a correlate of widespread cortical networks processing different aspects of emotionally arousing visual objects. In contrast, differences between affective categories in early gamma activity might reflect fast detection of aversive stimulus features. (C) 2001 Elsevier Science Ireland Ltd. All rights reserved.
\end{abstract}

Keywords: Gamma band; Event-related potentials; Emotion; Motivation; Perception; Human

\section{Introduction}

When an individual perceives a motivationally relevant stimulus, a variety of specific emotional processes emerge in the central and peripheral nervous system. It has been suggested that these processes are related to consummatory/appetitive or defensive/aversive action dispositions (e.g. Lang, 1994). This approach is associated with a twodimensional (2D) model of emotion: a valence dimension is related to variations on the level of the two motive systems, i.e. 'appetitive' versus 'aversive'. A second arousal dimension adds to the 2D affective space a component that modulates the emotional behavior with respect to activation or intensity (Lang et al., 1998a). Accordingly, modulatory neurophysiological processes have been suggested that mediate these behavioral changes in response to motivationally significant stimuli. In order to examine these modula-

\footnotetext{
* Corresponding author. Tel.: +49-7531-882696; fax: +49-7531882891.

E-mail address: andreas.keil@uni-konstanz.de (A. Keil).
}

tions on the level of cortical large-scale activity, viewing affective pictures has been suggested as a valid laboratory paradigm that allows for a systematic variation of affective stimulus characteristics (Diedrich et al., 1997; Ito et al., 1998; Cuthbert et al., 2000). The present study aimed at studying the brain mechanisms underlying human emotional processing by measuring induced gamma band activity (GBA) as well as event-related potentials (ERPs) in response to emotional pictures presented to one visual halffield, i.e. entering through one hemisphere.

Central nervous correlates of affective picture processing have been investigated using a variety of recording techniques and experimental designs (see Cacioppo and Gardner, 1999 for a review). Several authors studied ERPs associated with picture stimuli depicting scenes differing as to their affective characteristics (Mini et al., 1996; Palomba et al., 1997; Cuthbert et al., 1998; Ito et al., 1998; Cuthbert et al., 2000; Schupp et al., 2000). These studies have converged showing a sustained late positive wave ( $>300 \mathrm{~ms}$ latency) in response to emotionally arousing stimuli, which is attenuated when subjects are presented with calm or neutral 
pictures. Typically, the ERP differences observed in these studies lasted for several seconds, reflecting the duration of picture presentation. For example, Cuthbert et al. (2000) reported ERP differences between arousing and calm pictures in time ranges up to $5000 \mathrm{~ms}$ post-stimulus, with affective pictures being presented for $6000 \mathrm{~ms}$. A further line of research examined metabolic and blood flow measures of the human brain during viewing emotional stimuli. For example, viewing affectively arousing stimuli has been reported to be associated with activation in the bilateral amygdaloid complex (Schneider et al., 1995, 1997) medial pre-frontal cortex, thalamus, hypothalamus and midbrain (Lane et al., 1997b), anterior cingulate (Lane et al., 1997a, 1998), anterior temporal cortex, amygdala and hippocampal formation (Reiman et al., 1997), orbitofrontal cortex (Royet et al., 2000), left pre-frontal cortex, and fusiform gyrus (Dolan et al., 1996). In a study designed to investigate emotion-specific activation in visual cortex, functional magnetic resonance imaging (fMRI) revealed differential activation of regions in visual cortex in response to emotional as compared to neutral pictures that was most pronounced in right occipital areas (Lang et al., 1998b). Taken together, this evidence supports the idea of widespread networks that are involved in the variety of subprocesses associated with affective perception and behavior. For instance, afferent input from higher-order visual cortex and frontal cortex has been suggested as a possible mechanism for emotion-dependent changes in visual cortices (Mesulam, 1998). Distributed networks may thus modulate early stages of visual processing, paralleling results from studies of spatial selective attention (see Hillyard and Anllo-Vento, 1998 for a review).

One possible correlate of the integrated activity of distributed neuronal networks is the induced GBA (Engel et al., 1997; Singer et al., 1997). The term 'induced GBA' refers to those oscillations in electrophysiological recordings that lie in the higher frequency range of the temporal spectrum, typically above $20 \mathrm{~Hz}$, and are not phase-locked to the onset of a stimulus (Tallon-Baudry and Bertrand, 1999). One group of theoretical approaches suggests that synchronous responses of grouped cells including their timing is related to the representation of visual objects and scenes (Milner, 1974; Damasio, 1989). Comparisons between studies in humans and monkeys have indicated that the GBA can be recorded using traditional electrophysiological techniques (Müller et al., 1996), although this remains debatable (Juergens et al., 1999). Recent reports in this area have demonstrated that the modulation of induced GBA in time windows between 200 and $400 \mathrm{~ms}$ following the onset of a stimulus is associated with perception of coherent visual objects (Basar-Eroglu et al., 1996; Müller et al., 1996, 1997; Keil et al., 1999; Tallon-Baudry and Bertrand, 1999) and may be a signature of active memory (Pulvermüller et al., 1999). Furthermore, enhanced GBA was observed when subjects were required to activate an object's internal representation during the delay of a short-term memory task
(Tallon-Baudry et al., 1998), thus suggesting that a temporal correlation of high-frequency neuronal activity also takes place in top-down processing of visual objects. It has been demonstrated that GBA is also modulated by spatial selective attention, lending further support to its functional relevance (Gruber et al., 1999). Regarding emotional picture viewing, GBA at right-hemisphere electrodes showed a selective enhancement for affectively arousing, compared to neutral pictures when long viewing periods (i.e. 6000 ms) were analyzed (Müller et al., 1999).

In addition to induced high-frequency oscillations, several authors have described an early $(80-100 \mathrm{~ms}$ following onset of a visual stimulus) oscillatory response in the gamma range (Sannita et al., 1999) that has been referred to as 'evoked gamma response' (Galambos, 1992), and has been described as being phase-locked to the onset of a stimulus (Herrmann et al., 1999). Experimental evidence for possible correlates of this early GBA is, however, not as conclusive as for the induced GBA. For instance, while Herrmann et al. (1999) reported enhanced early gamma in response to coherent visual stimuli, other authors did not report such alterations (Tallon et al., 1995). The relation between early-evoked gamma and stimulus parameters therefore remains debatable.

Among other issues, one question that has been raised in terms of the central nervous mechanisms mediating affective stimulus concerns their asymmetric organization in the cerebral hemispheres (Tucker, 1984; Davidson, 1992). In extending our previous study, we employed a hemifield presentation paradigm in order to explore hemispheric differences in affective visual processing. Presentation of stimuli to the visual hemifields is a technique that has extensively been used in studies of visual spatial attention (e.g. Luck et al., 1990; Mangun and Buck, 1998). The advantages of this procedure are manifold: first, it is possible to experimentally manipulate the cerebral hemisphere that is first involved in processing of a stimulus presented in the contralateral visual hemifield. A second benefit, especially for studies of high-frequency brain activity, lies in the fact that lateralized processing as indicated by GBA can be distinguished more easily from electromyographic (EMG) artifacts that are unlikely to occur selectively in the hemisphere contralateral to the stimulus. Furthermore, differential response timing in the hemispheres, operationalized as the latencies of ERP peaks or spectral events, may provide important information regarding the processing steps within and between cerebral hemispheres (Hillyard and AnlloVento, 1998). Recently, this approach has been shown to be useful for the study of GBA modulation by visual selective spatial attention (Gruber et al., 1999). Although hemifield paradigms have substantially increased our understanding as to how lateralized networks mediate attentional processes, this technique has rarely been used in emotion research (Kayser et al., 1997; Pizzagalli et al., 1999). For example, Kayser et al. (1997) found effects of emotional content on ERP components N2, early P3, late 
P3, and slow wave, using unpleasant (dermatological disease) and neutral pictures. Asymmetries were reported for N2 and early P3, with maximal effects over the right parietal region. The N2-P3 amplitude was increased for negative compared to neutral stimuli at right-hemisphere recording sites.

In the present study, the view was adopted that the affective/motivational significance of a stimulus for the organism may result in phasic changes of human 'motivated attention' (Lang et al., 1990). Accordingly, it has been hypothesized that an individual's visual system may adapt to short-term requirements of external stimuli, depending on their aversiveness/pleasantness or importance to the organism (Lang et al., 1997). The experimental design of the present study allows an investigation into hemifield-related ERP and GBA modulations that vary along affective dimensions. In order to obtain near-optimal spatial sampling of electrocortical activity, we used a dense array electrode montage consisting of 129 electrodes. Posterior electrode clusters were used to test for category-specific modulation of visual processing. In extending previous work, the focus here was on the time course of parameters being sensitive to visual processing of visual stimuli. To this end, we used wavelet analysis techniques designed to enhance sensitivity to temporal changes in higher bands, compared to Fourier algorithms. In addition, anterior sensors were examined to test for anterior asymmetries as have been proposed e.g. by Davidson and coworkers (e.g. Davidson, 1999). Based on the work reviewed above, we expected a right-hemisphere preponderance of late ERP components and GBA modulations, with greater effects of affective parameters during picture viewing in the left visual field.

\section{Methods}

\subsection{Participants}

Ten right-handed volunteers ( 7 women, 3 men; age range 22-40 years, mean age 26.1) with normal or corrected-tonormal vision consented to participate. They received class credits or a small financial bonus (DM 20) for participating.

\subsection{Stimuli and procedure}

Sixty stimuli were selected from the International Affective Picture System (IAPS; CSEA, 1999) 3 picture categories were used differing in affective valence (pleasant, neutral, and unpleasant). Twenty pleasant pictures showed family and erotic scenes, 20 neutral pictures showed persons and household objects, and 20 unpleasant pictures depicted mutilation and attack scenes. The IAPS numbers of the stimuli are given in Appendix A. All pictures were presented on a 19 in. computer monitor with a refresh rate of $70 \mathrm{~Hz}$. Pictures subtended a visual angle of $8^{\circ}$ horizontally, the eccentricity of the center of the pictures to either side being $2.8^{\circ}$. The distance between the screen and the subjects' eyes was $1.7 \mathrm{~m}$. A chin rest was used in order to keep these parameters constant within and between participants. Pictures were presented for $1000 \mathrm{~ms}$, with an interstimulus interval that was randomly varying between 3000 and $6000 \mathrm{~ms}$. A fixation point was marked in the center of the screen. The order of presentation was pseudo-randomized both regarding hemifield and affective category. A sequence of more than two pictures of the same sub-category (e.g. mutilation, family, etc.) was prevented. Two blocks of 300 pictures were presented, resulting in a total of 100 trials per affective category/hemifield combination. Subjects were first presented with two examples of the affective stimuli for each affective category, respectively, that were not part of the experimental series. They were also instructed to maintain gaze on the fixation point and to avoid exploratory eye movements and eye blinks during picture presentation. These requirements were practiced until the participants were familiar with the procedure. Subsequently, the electrode net was applied and participants entered an electrically shielded chamber, where the electroencephalographic (EEG) recordings were conducted. After the EEG recordings, subjects viewed the 60 different pictures again in a pseudo-randomized order and were asked to rate the respective picture on two categories, affective valence and arousal, using a paper and pencil version of the self-assessment manikin (SAM; Bradley and Lang, 1994). In this last block, no hemifield presentation was done and subjects viewed each picture without being constrained in any way.

\subsection{Electrophysiological recordings}

EEG recordings were made using an EGI 128-channel system.(Electrical Geodesics, 1998). The vertex (recording site $\mathrm{Cz}$ ) was chosen as reference. As suggested for the Electrical Geodesics high input impedance amplifier, impedances were kept below $50 \mathrm{k} \Omega$. Data were sampled at 500 $\mathrm{Hz}$. All channels were pre-processed online by means of 0.1 $\mathrm{Hz}$ high- and $200 \mathrm{~Hz}$ low-pass filtering. Artifact-free epochs (300 ms pre-onset and $724 \mathrm{~ms}$ post-onset of the stimulus) were obtained using the procedure by Junghöfer et al. (2000). This procedure uses the distribution of trial statistics across single trials as an index of data quality and replaces sensors that are contaminated with artifact by spherical spline-interpolation. The standard deviation (SD) of the voltage for each time point across trials and participants is computed to assess the stability of the averaged waveform. For all subsequent analyses, we used the average reference. Horizontal (hEOG) and vertical electro-oculogram (vEOG) were computed from net electrodes that were sited at the outer canthi of both eyes and below and above the center of the right eye, respectively. The mean voltage of a $200 \mathrm{~ms}$ pre-stimulus baseline was subtracted to correct for offsets. After artifact correction, the mean number of trials remaining for each affective category/hemifield combination was 
67 , with a minimum value of 45 and a maximum value of 89 artifact-free trials.

\subsection{Data reduction and analysis}

\subsubsection{Data analysis $G B A$}

Spectral responses to affective pictures were examined using wavelet transform of the artifact-free epochs. The present procedure has been proposed by Bertrand et al. (1994) and is described in detail in the respective publications, e.g. by Tallon-Baudry et al. (1997, 1998)). In brief, complex Morlet wavelets $g$ can be generated in the time domain for different analysis frequencies $f_{0}$ according to

$g\left(t, f_{0}\right)=A^{\prime} \mathrm{e}^{-\left(t^{2} / 2 \sigma_{t}^{2}\right)} \mathrm{e}^{2 \mathrm{i} \pi f_{0} t}$

with $A^{\prime}$ depending on the parameter $\sigma_{f}$, specifying the width of the wavelet in the frequency domain, the analysis frequency $f_{0}$ and the user-selected ratio $m$ :

$A^{\prime}=\sigma_{f} \sqrt{2 \pi^{3}} \sqrt{\frac{m}{f_{0} \sqrt{\pi}}}$

with

$m=\frac{f_{0}}{\sigma_{f}}$

Thus, given a constant ratio $m$, the width of the wavelets in the frequency domain $\sigma_{f}$ changes as a function of the analysis frequency $f_{0}$. Wavelets of this family were normalized in order to have equal amounts of energy. For each epoch, time-varying spectral power in a given frequency band was obtained as the absolute value of the convolution of the signal with the wavelet for each epoch and complex spectra. Complex spectra were averaged response-locked across epochs before obtaining the absolute value (modulus). Likewise, averages across time windows and frequency bands were obtained from complex values, before computing the modulus. A constant $m=f_{0} / \sigma_{f}=7$ was used in order to obtain appropriate time and frequency resolution with wavelet center frequencies ranging from 9.8 to 68.8 Hz. For instance, the $41 \mathrm{~Hz}$ wavelet was characterized by an $\mathrm{SD}$ of $5.8 \mathrm{~Hz}$ in the frequency domain and a SD of $27 \mathrm{~ms}$ in the time domain, with higher frequency being related with increased temporal but lower frequency specificity $(30 \mathrm{~Hz}$ wavelet: SDs $=4.2 \mathrm{~Hz}, 38 \mathrm{~ms}$ ). Phase-locking of oscillatory activity was measured using the phase-locking factor proposed by Tallon-Baudry et al. $(1997,1998)$ ). This is a measure for phase identity across trials and is bounded between 0 (non-phase-locked signal) and 1 (phase-locked signal). Its statistical significance can be assessed by means of a circular statistic (Rayleigh test). A $250 \mathrm{~ms}$ pre-stimulus period $(-300$ to $-50 \mathrm{~ms}$ ) was used as baseline for the timefrequency information and the mean of this time window was subtracted from the time-frequency matrix for each frequency and time point. After wavelet transform, spectral power in 6 frequency bands was obtained by computing the average power for alpha $(9-13 \mathrm{~Hz})$, beta $(14-20 \mathrm{~Hz})$, low gamma $(21-30 \mathrm{~Hz})$, mid gamma $(31-45 \mathrm{~Hz})$, and high gamma $(46-65 \mathrm{~Hz})$. The $44.9 \mathrm{~Hz}$ wavelet $(\mathrm{SD}=6.4 \mathrm{~Hz})$ was excluded from these averages to avoid excessive overlap between the mid and high gamma bands. Four time windows were examined that were selected on the basis of grand mean time-frequency distributions to contain the most pronounced spectral changes. (1) An early window, ranging from 70 to $90 \mathrm{~ms}$ after stimulus onset, (2) a 170 $220 \mathrm{~ms}$ window, (3) a 280-340 ms window, and (4) a late window ranging from 480 to $550 \mathrm{~ms}$. Since Müller et al. (1999) have reported GBA effects of affective picture viewing using longer presentation times and time windows with low temporal specificity, the focus of the present study was on earlier GBA/ERP modulations in the visual system and their temporal dynamics. For statistical analysis, mean values for these windows were averaged across electrodes into regional means. Four electrode clusters were used to account for the hemifield stimulation and to be sensitive for signals both related to posterior and to anterior sources.

Fig. 1 displays the relative position of the selected clusters with respect to sites of the international 10-20 system. The mean spectral power within each frequency band and time window was subject to 4 factor repeated-measure analysis of variance (ANOVA) with the factors being HEMIFIELD (left, right), CATEGORY (pleasant, neutral, unpleasant), HEMISPHERE (left, right), and RECORDING SITE (temporal anterior, temporal posterior) for each frequency band, respectively. Effects of picture categories on SAM scores were evaluated using repeated measurement ANOVA with the factor CATEGORY (pleasant, neutral, unpleasant).

\subsection{Data analysis ERP}

Two methods were employed for analysis of ERP data. First, after averaging separately according to the factorial structure of the experiment, 3 pronounced peaks were identified on the basis of grand means: a P1 deflection, peaking around $120 \mathrm{~ms}$, an $\mathrm{N} 1$ being maximal around $160 \mathrm{~ms}$ and a P3-like wave that showed latencies around $280 \mathrm{~ms}$ (see Table 1). Peak latencies and amplitudes of these 3 deflections were extracted using the time windows identified on the basis of grand average data. For this purpose, voltages and latencies at electrodes corresponding to the sites $\mathrm{T} 5$ and $\mathrm{T} 6$ of the international 10-20 system were examined, where the visual ERP peaks showed maximum amplitude. Thus, a $2 \times 3 \times 2$ ANOVA was computed for each parameter of each component, having the within-subjects factors HEMIFIELD (left, right), CATEGORY (pleasant, neutral, unpleasant), and SITE (T5, T6). This procedure allowed for investigating the latencies, which may reveal differences in response timing depending on affective valence or arousal.

A second approach used ANOVAs on regional voltage means for data reduction. Based on grand mean traces and inter-individual peak variability, time windows for the 5 major deflections were identified: P1 (100-130 ms), N1 


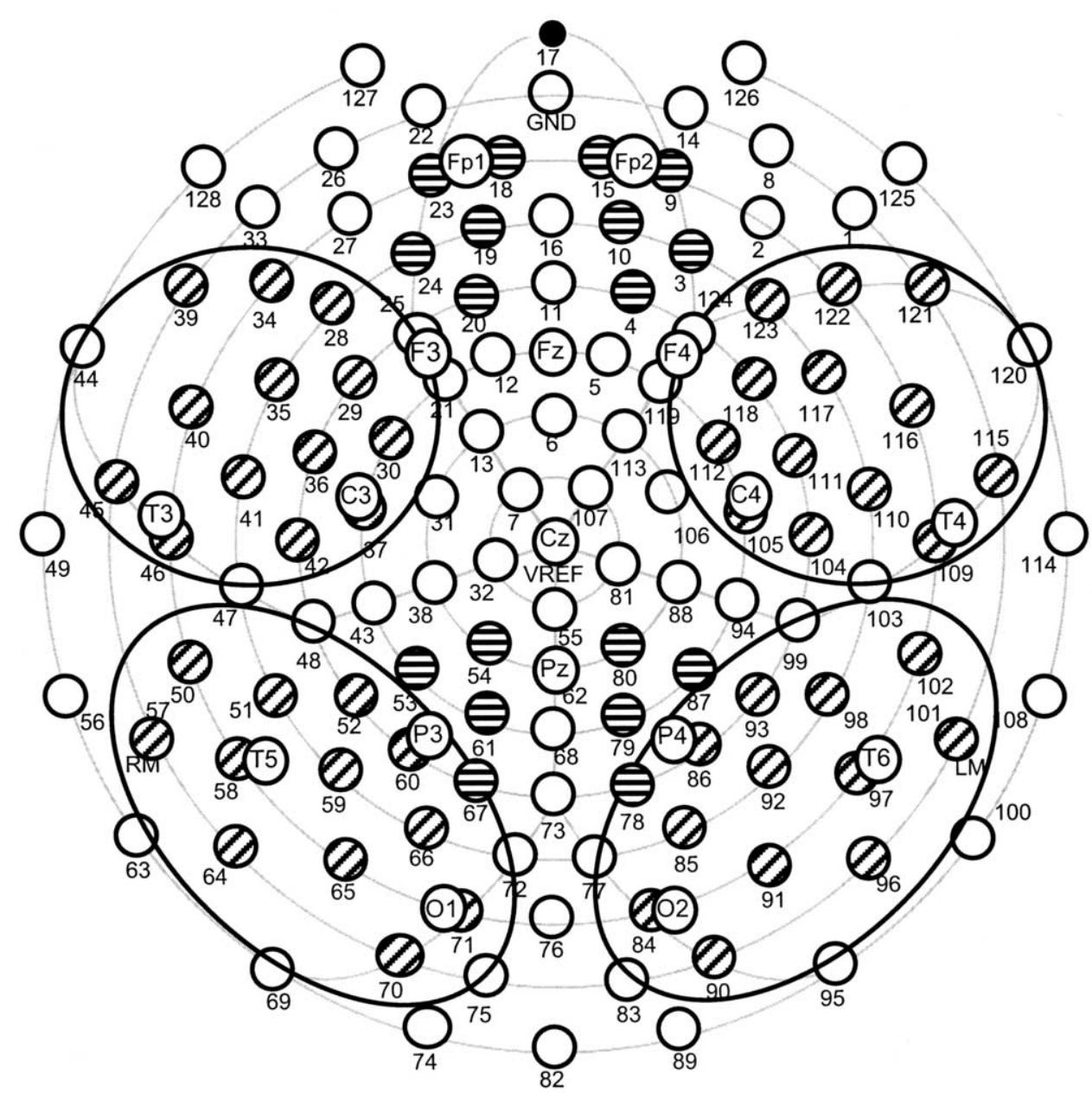

Fig. 1. Layout of electrodes showing sites of the 128 sensors relative to right mastoid (RM), left mastoid (LM), and sites from the international 10-20 system. Encircled sites: regions used for ANOVAs on spectral measures. Posterior encircled sites also served for statistical testing of P1, N1, and P3. Hatched electrodes around F3, Fp1/F4, Fp2, and P3/P4 were grouped to test significance of anterior N2 and late positive wave.

(155-185 ms), P3 (280-340 ms), anterior N2 (220-260 ms), and a late positive wave (400-600 ms). In order to achieve comparability with respect to GBA analyses, ANOVAs on mean voltages of ERPs were computed for the identical set of electrode clusters as described above for GBA analysis. Appropriateness of this procedure for detecting voltage maxima of respective components was controlled by means of grand mean topographical distributions. Furthermore, effects of experimental manipulations on anterior N2 and late positive wave were further tested in hemispheresymmetrical clusters formed around anterior (Fp1, Fp2 for anterior N2) and parieto-occipital (P3, P4 for late positive wave) recording sites, where these components showed maximal amplitude. The respective electrode clusters and time windows are shown in Fig. 1. Mean voltages obtained in the relevant time windows and electrode groups were subject to $2 \times 3 \times 2$-ANOVA with the within factors HEMIFIELD (left, right), CATEGORY (pleasant, neutral unpleasant), and HEMISPHERE (left, right). Significant main effects and interactions were followed-up using Scheffé tests, where appropriate.

\section{Results}

\subsection{SAM ratings}

As expected, the ANOVAs on the SAM ratings for emotional valence revealed a significant effect of CATE-

Table 1

Mean peak voltages $(\mu \mathrm{V})$ and latencies $(\mathrm{ms}) \pm$ SDs for the P1, N1, and P3

\begin{tabular}{lccc}
\hline & $\mathrm{P} 1$ & $\mathrm{~N} 1$ & $\mathrm{P} 300$ \\
\hline $\begin{array}{l}\text { Mean peak amplitude } \\
(\mu \mathrm{V}) \text { contralateral } \pm \mathrm{SD}\end{array}$ & $2.41 \pm 1.45$ & $-1.89 \pm 1.5$ & $2.19 \pm 1.89$ \\
$\begin{array}{l}\text { Mean peak latency (ms) } \\
\text { contralateral } \pm \text { SD }\end{array}$ & $114 \pm 19$ & $174 \pm 20$ & $247 \pm 31$ \\
$\begin{array}{l}\text { Mean peak amplitude } \\
(\mu \mathrm{V}) \text { ipsilateral } \pm \text { SD }\end{array}$ & $1.32 \pm 1.2$ & $-1.79 \pm 1.71$ & $1.34 \pm 1.48$ \\
$\begin{array}{l}\text { Mean peak latency (ms) } \\
\text { ipsilateral } \pm \text { SD }\end{array}$ & $137 \pm 26$ & $195 \pm 24$ & $255 \pm 29$ \\
\hline
\end{tabular}

${ }^{\text {a }}$ Values were obtained at electrodes 58 and 97 , corresponding to sites T5 and $\mathrm{T} 6$ of the international 10-20 system. Means reflect an average across 10 participants. 
GORY $(F(2,18)=113.8 ; \quad P<0.01)$. Post hoc testing demonstrated that this effect was due to significant differences between each of the 3 picture categories $(P<0.01)$. Thus, pictures were experienced according to their respective category. Likewise, the ratings on arousal exhibited significant differences between categories $(F(2,18)=95.2$; $P<0.01)$. Post hoc testing revealed that affective pictures were associated with higher arousal than neutral pictures $(P<0.01)$. In addition, arousal was rated significantly higher for pleasant as compared to neutral pictures $(P<0.01)$, lower for pleasant as compared to unpleasant $(P<0.01)$, and lower for neutral as compared to unpleasant slides $(P<0.01)$.

\subsection{Results $G B A$}

As is illustrated by the grand mean contour plots of right parietal electrodes shown in Fig. 2, hemifield presentation of
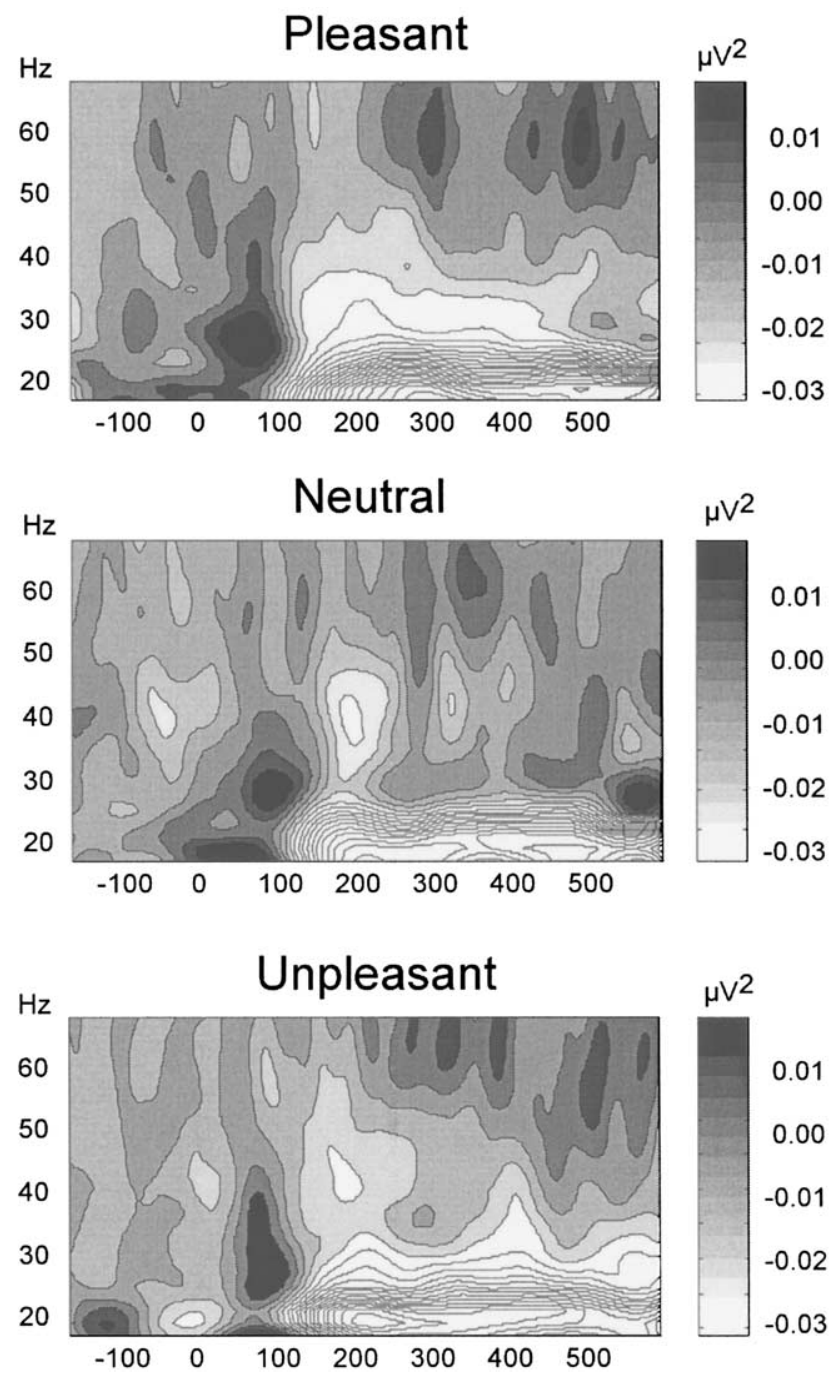

Fig. 2. Grand mean time-frequency contour plots $(N=10)$ of changes in the spectral power in response to affective pictures presented to the left visual field, averaged across the right posterior electrodes used for statistical analysis (see Fig. 1). affective pictures was associated with a pronounced broadband amplitude decrease in the frequency range below 20 $\mathrm{Hz}$. The higher bands displayed an increase as compared to baseline level that was most pronounced in the range above $50 \mathrm{~Hz}$. Results for ANOVAs on the mean spectral power for the 5 selected frequency ranges and 4 time windows are reported below.

\subsection{Alpha band}

A main effect of HEMIFIELD $(F(1,9)=7.1 ; P<0.05)$ was found in the early window (70-90 ms), showing that presentation in the left hemifield was associated with greater decrease of alpha power than presentation in the right hemifield. Accordingly, alpha reduction in the second window (180-220 ms) was enhanced at right posterior recording sites $(F(1,9)=12.9 ; P<0.01)$. However, alpha power did not discriminate between affective categories in the first two time ranges. In contrast, after $280 \mathrm{~ms}$ (280-340 $\mathrm{ms}$ ), alpha power displayed a significant HEMIFIELD $\times$ CATEGORY interaction $(F(2,18)=4.5 ; P<0.05)$, showing that right hemifield presentation was associated with a greater decrease of alpha selectively for unpleasant as compared to both pleasant and neutral pictures. The left hemifield presentation had the opposite effect, being associated with selective alpha decrease in response to pleasant pictures. Neutral pictures showed no sensitivity to the respective hemifield, whereas pleasant and unpleasant pictures showed reversed patterns of alpha reduction, depending on stimulus location. Again, right posterior recording sites contributed most to the spectral power in this time-frequency range $(F(1,9)=12.3 ; P<0.01)$. Neither main effects nor interactions involving CATEGORY were found in the $500 \mathrm{~ms}$ window. Paralleling results from 200 to $300 \mathrm{~ms}$ windows, the significant interaction HEMISPHERE $\times$ SITE revealed that posterior right sites contributed most to the spectral power reduction across conditions and hemifields $(F(1,9)=8.8 ; P<0.05)$.

\subsection{Beta band}

Throughout time ranges, no significant effects of picture category were observed. Post hoc testing of the significant interactions HEMIFIELD $\times$ HEMISPHERE for the 200 and $300 \mathrm{~ms}$ windows $\left(F_{\mathrm{s}}(1,9)=13.6,6.9 ; P_{\mathrm{s}}<0.01\right)$, indicated that only left hemifield presentation was related to stronger beta decrease in the contralateral hemifield (post hoc $P_{\mathrm{s}}<0.05$ ). In contrast, presentation on the right did not result in asymmetrical beta topography.

\subsection{Low gamma band}

In the low gamma band $(20-30 \mathrm{~Hz})$, there was a main effect of SITE in the 3 late time windows, showing that decrease as compared to baseline was stronger at posterior than anterior sites (post hoc $P_{\mathrm{s}}<0.05$ ). Furthermore, this posterior reduction was most pronounced at right-hemi- 
sphere sites (post hoc $P_{\mathrm{s}}<0.05$ ). Right-hemisphere lower GBA in the $200 \mathrm{~ms}$ range showed a modulation according to affective picture category (CATEGORY $\times$ HEMISPHERE: $F(2,18)=4.00 ; P<0.05)$. This effect was due to a stronger decrease of right-hemisphere lower GBA when unpleasant pictures as compared to neutral pictures were viewed (post hoc $P<0.05$ ). Thus, this modulation occurred independently of HEMIFIELD. No effect was found for the early window.

\subsection{Mid gamma band}

While all the lower bands showed a relative decrease in spectral power across conditions and time windows, mid gamma $(30-45 \mathrm{~Hz})$ was enhanced as compared to baseline in the (70-90 ms) window. The phase-locking factor computed for this time-frequency window $(R=0.11)$ reached statistical significance $(P<0.05)$. This early response showed a main effect of CATEGORY $(F(2,18)=4.0 ; P<0.05)$, with higher increase across sites when unpleasant pictures were presented in any of the hemifields (post hoc $P<0.05$; see Fig. 3, top). Accordingly, the subsequent $(200 \mathrm{~ms})$ power decrease below baseline level was less pronounced for unpleasant pictures (CATEGORY: $\quad F(2,18)=4.1 ; \quad P<0.05 ; \quad$ post hoc $P<0.05)$. However, this modulation was restricted to early processing. No comparable effects were found for the later windows.

\subsection{High gamma band}

In the high gamma range $(45-65 \mathrm{~Hz})$, effects were restricted to the $500 \mathrm{~ms}$ time window: a significant interaction CATEGORY $\times$ HEMISPHERE $\times \operatorname{SITE}(F(2,18)=4.0$; $P<0.05)$ revealed that posterior right sites exhibited an affect modulation, with higher spectral power being related to viewing of both pleasant and unpleasant, compared to neutral pictures. Additionally, the significant 4-way interaction HEMIFIELD $\times$ CATEGORY $\times$ HEMISPHERE $\times$ SITE $(F(2,18)=8.8 ; P<0.01)$ revealed that arousal modulation in the right hemisphere was due to the effect of left hemifield presentation of emotive, compared to neutral pictures (post hoc $P_{\mathrm{s}}<0.05$; see Fig. 3, bottom). Phase-locking factors did not reach statistical significance in this timefrequency range (maximum $R=0.03$ ).

\subsection{Results ERP}

\subsubsection{Peak amplitudes and latencies}

Table 1 shows the mean peak voltages and latencies including SDs of $\mathrm{P} 1, \mathrm{~N} 1$, and $\mathrm{P} 3$ peaks. These were obtained at sites corresponding to T5 and T6 of the international 1020 system where these deflections were most pronounced. It is evident that across picture categories $\mathrm{P} 1$ and $\mathrm{N} 1$ were larger in the contralateral hemisphere, respectively, and exhibited longer latencies in the ipsilateral hemisphere (see Fig. 4). While vertical EOG showed no systematic time-locked activity, horizontal EOG indicated small stimulus-locked saccades to the side of the stimulus. These EOG responses started at $120 \mathrm{~ms}$ post-stimulus and were maintained for $60-80 \mathrm{~ms}$. ANOVAs on peak amplitudes and latencies showed the following results:

P1 amplitudes were significantly higher when stimuli were presented in the left as compared to the right hemifield, across conditions and sites $(F(1,9)=5.9 ; P<0.05)$. In addition, a HEMIFIELD $\times$ SITE interaction $(F(1,9)=10.9 ; P<0.01)$ indicated higher amplitudes in the contralateral hemisphere, respectively. P1 amplitude enhancement in response to affective as compared to neutral stimuli across hemifields and sites did not reach statistical significance $(F(2,18)=3.8$; $P=0.06$ ). A different picture emerged for the N1. A main effect of CATEGORY $(F(2,18)=4.0 ; P=0.05)$ showed a modulation of the $\mathrm{N} 1$ amplitude, being enhanced for emotive pictures selectively, across hemifields and sites. Furthermore, this effect was shown to be restricted to left hemifield presentation $($ CATEGORY $\times$ HEMIFIELD: $F(2,18)=3.9$; $P<0.05)$. The $\mathrm{P} 3$ peak was larger in the contralateral hemisphere (HEMIFIELD $\times$ SITE: $F(1,9)=8.7 ; P<0.05$ ), but did not show further effects.

As expected, the P1 peak showed delayed peak latencies in the ipsilateral as compared to the contralateral hemisphere $(F(1,9)=65.4 ; P<0.01)$. However, P1 latencies were not sensitive to affective stimulus properties. In contrast, the $\mathrm{N} 1$ component, besides showing laterality effects $(F(1,9)=52.2 ; \quad P<0.01)$, was delayed when emotive as compared to neutral pictures were presented $(F(2,18)=4.44 ; P<0.05)$, whereas pleasant and unpleasant pictures did not differ. No significant effect was seen in the P3 latencies.

\subsubsection{Regional means}

ANOVAs on mean voltages revealed that across hemifields and hemispheres both the mean P1 and N1 amplitude in the posterior clusters were significantly higher for affective than for neutral pictures $(F(2,18)=5.2,4.3$; $P<0.05$, post hoc $\left.P_{\mathrm{s}}<0.05\right)$. P1 and $\mathrm{N} 1$ regional means did not change as a function of hemifield or hemisphere, nor was there any further significant interaction including CATEGORY. The anterior negative deflection peaking at $250 \mathrm{~ms}$ (N2a) was enhanced selectively in response to neutral pictures whereas arousing pictures showed smaller $\mathrm{N} 2 \mathrm{a}$ amplitudes $(F(2,18)=4.2$; $P<0.05)$. Again, hemifield and hemisphere had no effect on this phenomenon. In contrast, the $\mathrm{P} 3$ wave at posterior sites, which temporally co-occurred with the N2a, was not modulated as a function of emotional properties. Finally, the slow positive deflection starting at $400 \mathrm{~ms}$ post-stimulus exhibited a significant effect of emotional arousal at posterior sites $(F(2,18)=3.5 ; P<0.05)$, with enhanced regional means during viewing of pleasant and unpleasant pictures, compared to neutral pictures (post hoc $\left.P_{\mathrm{s}}<0.05\right)$. 


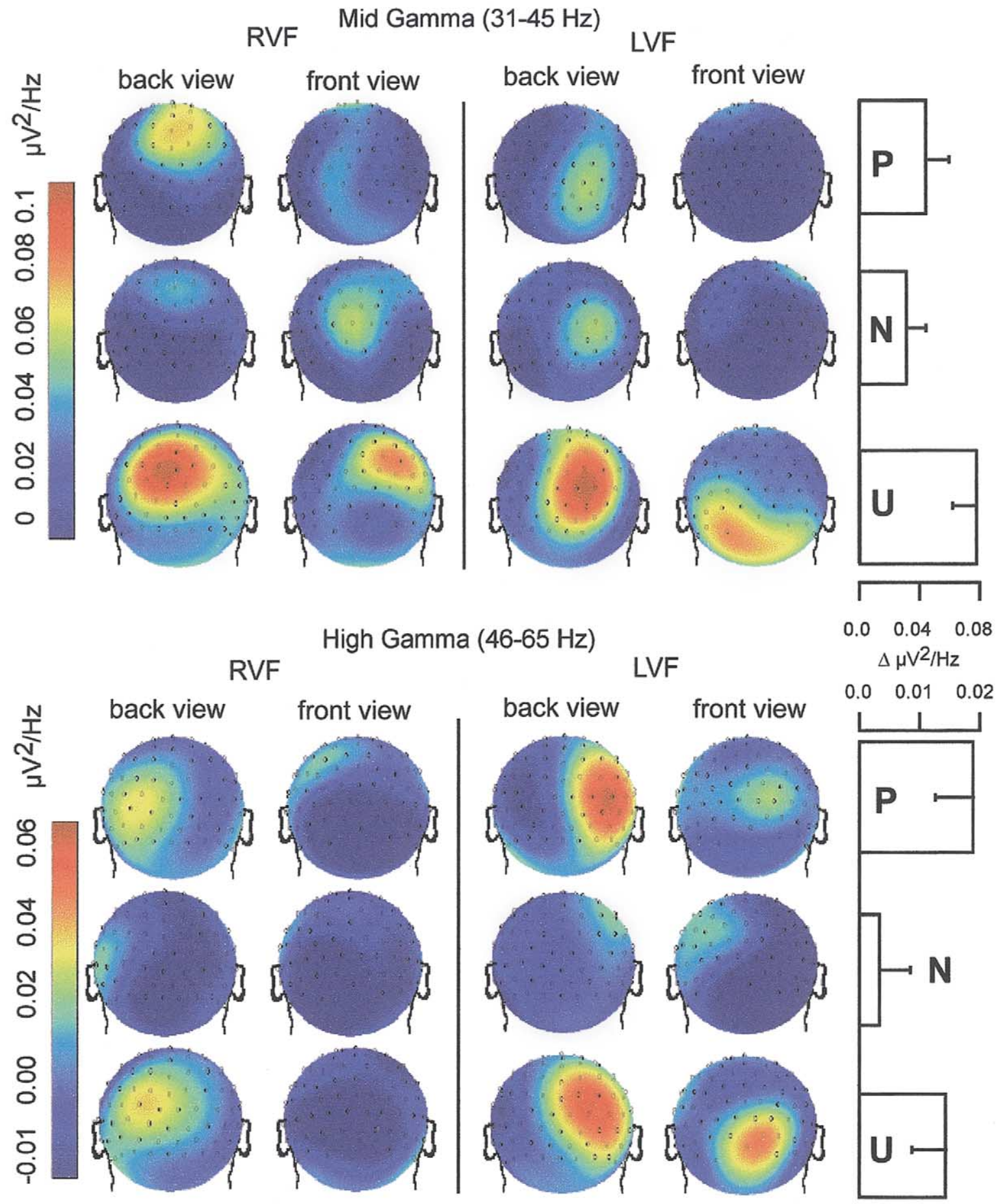

Fig. 3. Top panel: grand mean topographical distribution $(N=10)$ of power changes in the mid gamma band during the early time window $(70-90$ ms) in response to the 3 picture categories Pleasant $(\mathrm{P})$, Neutral $(\mathrm{N})$, Unpleasant $(\mathrm{U})$, presented to the left (LVF) or right visual field (RVF). Bottom panel: grand mean topographical distribution $(N=10)$ of the changes in the high gamma band in response to the 3 picture categories presented to the left (LVF) or right visual field (RVF) during the late time window (480-550 ms). Right-hand bar plots show the mean power changes for the electrodes used for statistical analysis. Error bars indicate standard error.

\section{Discussion}

\subsection{GBA power modulation}

Two salient effects in terms of GBA modulation were found in this study: first, early GBA in the range around 30 $\mathrm{Hz}$ showing its peak at a latency of $80 \mathrm{~ms}$ post-stimulus discriminated between unpleasant stimuli and other stimulus categories. Second, a late $(480-550 \mathrm{~ms})$ increase in the higher gamma range was significantly higher for affectively 

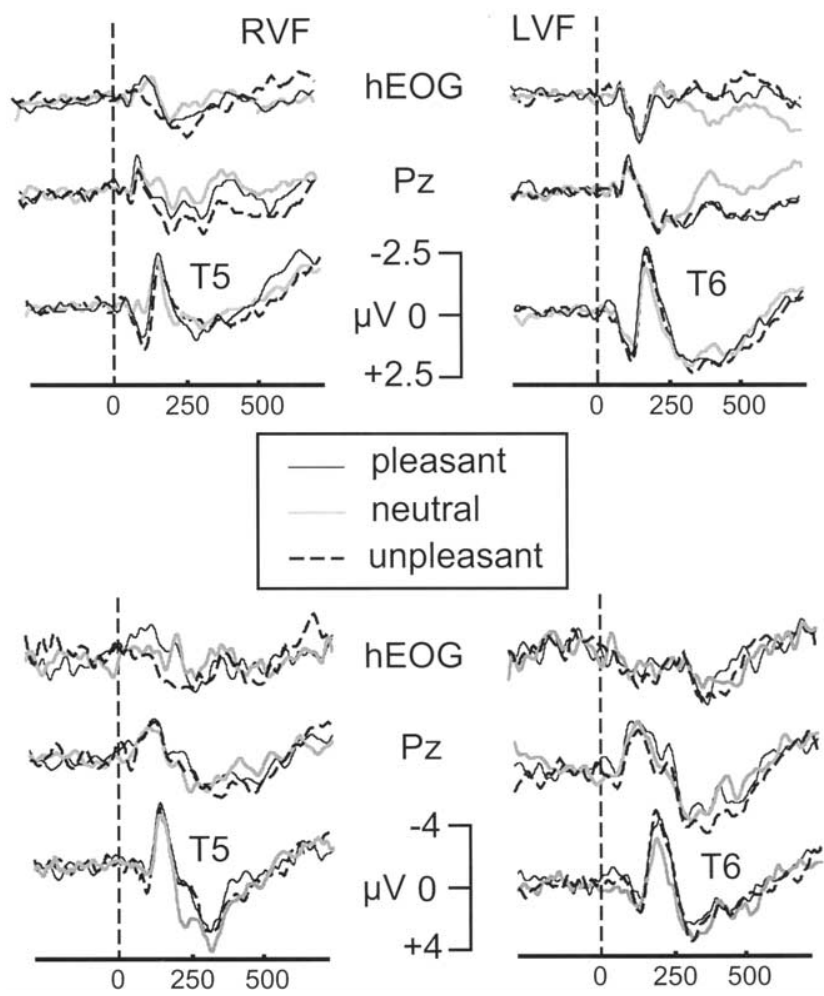

Fig. 4. Top panel: grand mean ERP waveforms $(N=10)$ of hEOG, and at electrode sites corresponding to $\mathrm{Pz}, \mathrm{T} 6$, and T5, respectively, in response to the 3 picture categories presented to the left (LVF) or right visual field (RVF). Bottom panel: waveforms for a representative participant.

arousing than for neutral pictures. This modulation was seen at temporo-occipital recording sites, being most pronounced on the right hemisphere, across hemifields. Thus, this latter effect regarding right-hemispheric modulation of GBA is in line with a previous study of GBA modulation in emotional picture processing (Müller et al., 1999). However, the present study allows for a more detailed analysis of oscillatory brain activity at different latencies. For instance, we did not observe induced GBA alterations as a function of emotional arousal in the latency ranges prior to the $500 \mathrm{~ms}$ window. This is consistent with the view that higher-order visual cortices receive more dense afferents from other structures being involved in evaluation of the motivational significance, such as the amygdala (Rolls, 1995; Mesulam, 1998). Furthermore, the topographical distribution of this late gamma modulation suggests that emotional arousal is related to alterations in temporo-occipital cortices, as has been found in studies with blood flow measures (Lang et al., 1998b).

The present hemifield paradigm revealed that, although less pronounced, late GBA modulations (480-550 ms) were also found in the left hemisphere when stimuli were presented in the right visual hemifield. A general righthemisphere dominance of the late GBA across affective categories was found, which is consistent with GBA findings from attentional hemifield tasks (Gruber et al., 1999). Thus, an exclusive role of the right cerebral hemisphere in emotional processing is not supported by our data.
Time course and specific topography of affective GBA modulations during percept formation showed discrimination between early and late processing stages. The present finding of an induced GBA enhancement in the band above $45 \mathrm{~Hz}$ compared to baseline, starting at about $300 \mathrm{~ms}$ throughout conditions (see Fig. 2) is consistent with the literature in this area (Basar-Eroglu et al., 1996; Sannita, 2000). Usually, onset of these responses to coherent visual objects occurs in a window between 200 and $400 \mathrm{~ms}$ after onset of a stimulus (Tallon-Baudry and Bertrand, 1999). Interestingly, this 200-400 ms gamma response did not discriminate between affective categories.

We found a pronounced early oscillatory event that showed its peak at about $80-100 \mathrm{~ms}$ post-stimulus in the contralateral hemisphere. This early event has been described by other authors using visual stimuli (TallonBaudry et al., 1997; Herrmann et al., 1999). Usually, it is strongly phase-locked to the onset of the stimulus and is located in lower ranges of the gamma frequency band (Sannita et al., 1999). Recordings in animals as well neuromagnetic evidence in man suggest that this oscillatory response may be generated in primary visual cortical areas (Sannita et al., 1999). Given that the temporal half-width of the $30 \mathrm{~Hz}$ wavelet in this study is $38 \mathrm{~ms}$, it seems likely that this early response precedes the $\mathrm{P} 1$ component rather than co-occur with it. This response differed from early ERP in being sensitive to aversive stimulus material only, showing no discrimination between neutral and pleasant pictures. This pattern suggests that amplitude modulation of this early time-locked response reflects amplified processing of stimulus features characterizing aversive stimuli. It is, however, unclear which neuronal mechanism would mediate such early enhancement of the significant features.

Another measure of spectral changes in response to the affective stimuli, namely alpha reduction as compared to baseline, showed an interaction between stimulus location and affective valence. With stimuli presented in the right hemifield, alpha power showed a greater decrease of alpha selectively for unpleasant than for neutral and pleasant pictures. Left hemifield presentation had the opposite effect, being associated with higher alpha decrease in response to pleasant, compared to neutral and unpleasant pictures. Interestingly, the right hemisphere contributed most to this effect, across hemifields. No comparable results were seen in the gamma range. The relevance of alpha modulation has been discussed extensively in terms of general properties of the human brain (see e.g. Basar et al., 1997). Traditional accounts have tried to interpret alpha reduction in terms of desynchronization of neuronal activity, thus being related to enhanced activation in task-relevant circuitry (Shagass, 1972). Recent accounts in the literature view alpha as reflecting regional cortical idling states (Pfurtscheller et al., 1996). Anterior asymmetries in alpha reduction have been viewed as correlates of changes on individual affective states (Davidson, 1998). The present data provide evidence for the notion that alpha reduction and GBA enhancement 
measure different and complementary aspects of brain electric dynamics. Additionally, they do not appear to be merely inversely related. The wavelet family used in the present study was not designed to examine alpha modulation. However, the pronounced lateralization of the GBA to the contralateral hemisphere, as well as the sensitivity of GBA for phasic emotional changes seen in the current data, demonstrate that GBA measures may be a more specific indicator of affective modulation in visual processing than is alpha reduction.

\subsubsection{ERPs}

Two main results were seen regarding ERP measures. First, early ERP components, namely the P1 and N1 showed unspecific enhancement as a response to emotive, compared to calm pictures. Second, the late positive wave was found to be located at central parietal sites, showing neither lateralization nor sensitivity to the hemifield where a picture was presented. The fact that an early effect according to the pictures' arousal was observed is supportive for an early allocation of motivated attention sensu Lang (Lang, 1994; Lang et al., 1997) to stimuli with high arousal properties. Recent reports of early (80-116 ms) differentiation between liked and disliked facial stimuli give support to this view (Pizzagalli et al., 1999).

A well-established finding in studies of visual spatial attention paradigms has been an amplitude enhancement of $\mathrm{P} 1 / \mathrm{N} 1$ in response to stimuli presented to the attended hemifield, compared to non-attended locations in the field of view (Hillyard and Anllo-Vento, 1998). This enhancement has been repeatedly found to be more pronounced on the right than left hemisphere. Likewise, functional imaging studies have shown a right-hemisphere preponderance in attentional processes (Lang et al., 1998b). It is unclear however, whether these same principles apply to emotional picture viewing. If attentional resources are allocated according to emotional arousal/motivational relevance, the topographical pattern of ERP and GBA should show similar hemispheric differences as in studies of spatial selective attention. Given the similarity of the present findings to those obtained with attentional paradigms, future studies might examine the relationship between instructed and motivated attention and their effects on ERP measures.

Modulation of the late positive wave was found to show similar topography and time course as in previous work (Cuthbert et al., 2000). The topography of this component suggested a right parietal origin across hemifields. This can be considered supportive for a higher-level modulatory process involving parieto-occipital areas. Processes involving these regions have also been proposed as important for spatial attention (Corbetta, 1998; Mesulam, 1998). Thus, it may well be that brain structures mediating spatial awareness and attention overlap in part with those that moderate attention to objects with motivational significance. Additionally, a topographical dissociation between the P3 and the late positivity was achieved with the current design, showing that only the P3 amplitude was enhanced at contralateral sites, with a pronounced dependence of stimulus location. An experimental dissociation can be seen in the fact that only the late positive wave, but not the P3, was sensitive for emotional arousal.

In terms of methodology, one major question concerns the role of the lateral presentation and the effects of small time-locked saccades. While allowing for a more detailed visual analysis of the affective stimuli, presentation times of $1000 \mathrm{~ms}$ have the disadvantage that initial visual processing steps cannot be unambiguously attributed to the contralateral hemisphere only, after saccades have been initiated. In the present study, the observed averaged hEOG responses were small and both ERP and GBA measures showed a pronounced lateralization according to the hemifield procedure. We thus assume that we achieved a predominantly contralateral execution of important steps in visual processing. Furthermore, a direct contamination of results by eye artifact seems unlikely, given that the time window with hEOG shifts was not included in the ERP and GBA analyses.

We report converging experimental effects for the late ERP and GBA measures, but diverging findings for the early modulations. Furthermore, we found a lower GBA peak preceding the P1/N1 deflection of the ERP. Although the relationship between ERP and GBA measures is debatable, some evidence has suggested that early ERP reflects initial, time-locked activation of neuronal assemblies, whereas GBA may reflect subsequent oscillatory activity in the relevant cell assemblies (Pulvermüller, 1999). Cell assemblies contain neurons that may be widespread across cortical regions (Singer et al., 1997). They are characterized by strong internal connectivity and thus get fully activated when a sufficient fraction of members is reached. Once activated, the representation remains active for a short period. Accordingly, oscillatory GBA has been viewed as a signature of the activation in cell assemblies (Singer et al., 1997). According to this view, the first cortical EEG response would be produced by the ignition of the cell assembly that corresponds to the representation of the respective stimulus, for instance a snake or a chair. It has been reported that emotionally arousing representations are activated faster (Bradley et al., 1992; Murphy and Zajonc, 1993; Morris et al., 1999) and with greater response amplitude (Cuthbert et al., 2000). The early mid gamma response and the N1 amplitude may reflect this phenomenon. In a second processing step, the cortical network related to the stimulus concept might become activated, in the case of a snake or a spider, for instance, the related fear structure (Lang et al., 1997). This activation may in turn be reflected in the late GBA and late positive wave of the ERP.

In summary, the present data have shown that visual processing may be altered by affective stimulus properties at several processing stages, starting at the level of the P1/ N1 component of the ERP and at mid GBA responses at 80 $100 \mathrm{~ms}$ post-stimulus. Parameters of higher processing 
stages showed a modulation as function of emotional arousal, together with a topographical distribution that is consistent with generators in higher-order visual, as well as right parietal cortices. These findings are supportive for a righthemispheric dominance in emotional perception. Furthermore, they are in line with the notion that the integrated activity of distributed networks involved in emotional perception requires high-frequency neuronal mass activity within and between the relevant structures.

\section{Acknowledgements}

The authors would like to thank Ursula Lommen, Niklas Ihssen, and Heidi Messmer for help in data acquisition. Research was supported by the Deutsche Forschungsgemeinschaft.

\section{Appendix A. Appendix}

IAPS numbers of stimuli used in the present study:

Pleasant pictures: 2050, 2070, 2080, 2160, 2165, 2170, $2311,2340,2341,2360,4650,4651,4652,4658,4659$, 4660, 4664, 4670, 4680, 4690.

Neutral pictures: 2190, 2200, 2210, 2230, 2381, 2440, 2480, 2570, 2850, 7002, 7009, 7010, 7020, 7030, 7040, 7080, 7175, 7233, 7235, 9070 .

Unpleasant pictures: 1050, 1120, 1201, 1300, 1930, $3000,3010,3050,3060,3071,3080,3102,3110,3130$, $3530,6260,6350,6510,6540,9405$.

\section{References}

Basar E, Schurmann M, Basar-Eroglu C, Karakas S. Alpha oscillations in brain functioning: an integrative theory. Int J Psychophysiol 1997;26:529.

Basar-Eroglu C, Struber D, Schurmann M, Stadler M, Basar E. Gammaband responses in the brain: a short review of psychophysiological correlates and functional significance. Int $\mathbf{J}$ Psychophysiol 1996;24:101-112.

Bertrand O, Bohorquez J, Pernier J. Time-frequency digital filtering based on an invertible wavelet transform: an application to evoked potentials. IEEE Trans Biomed Eng 1994;41:77-88.

Bradley MM, Lang PJ. Measuring emotion: the Self-Assessment Manikin and the semantic differential. Journal of Behavior Therapy \& Experimental Psychiatry 1994;25(1):49-59.

Bradley MM, Greenwald MK, Petry MC, Lang PJ. Remembering pictures: pleasure and arousal in memory. J Exp Psychol Learn Mem Cogn 1992;18:379-390.

Cacioppo JT, Gardner WL. Emotion. Annu Rev Psychol 1999;50:191-214.

Corbetta M. Frontoparietal cortical networks for directing attention and the eye to visual locations: identical, independent, or overlapping neural systems? Proc Natl Acad Sci USA 1998;95:831-838.

CSEA: international affective picture system (IAPS): technical manual and affective ratings. Gainesville, FL: NIMH - Center for the study of emotion and attention, University of Florida, 1999.

Cuthbert BN, Schupp HT, Bradley M, McManis M, Lang PJ. Probing affective pictures: attended startle and tone probes. Psychophysiology 1998;35:344-347.
Cuthbert BN, Schupp HT, Bradley MM, Birbaumer N, Lang PJ. Brain potentials in affective picture processing: covariation with autonomic arousal and affective report. Biol Psychol 2000;52:95-111.

Damasio AR. Time-locked multiregional retroactivation: a systems-level proposal for the neural substrates of recall and recognition. Cognition 1989;33:25-62.

Davidson RJ. Anterior cerebral asymmetry and the nature of emotion. Brain Cogn 1992;20:125-151.

Davidson RJ. Anterior electrophysiological asymmetries, emotion, and depression: conceptual and methodological conundrums. Psychophysiology 1998;35:607-614.

Davidson RJ. The functional neuroanatomy of emotion and affective style. Trends Cogn Sci 1999;3:11-21.

Diedrich O, Naumann E, Maier S, Becker G. A frontal positive slow wave in the ERP associated with emotional slides. J Psychophysiol 1997;11:71-84.

Dolan RJ, Fletcher P, Morris J, Kapur N, Deakin JF, Frith CD. Neural activation during covert processing of positive emotional facial expressions. NeuroImage 1996;4:194-200.

Engel AK, Roelfsema PR, Fries P, Brecht M, Singer W. Role of the temporal domain for response selection and perceptual binding. Cereb Cortex 1997;7:571-582.

Galambos R. A comparison of certain gamma-band $(40 \mathrm{~Hz})$ brain rhythms in cat and man. In: Basar E, Bullock T, editors. Induced rhythms in the brain, Berlin: Springer, 1992. pp. 103-122.

Gruber T, Müller MM, Keil A, Elbert T. Selective visual-spatial attention alters induced gamma band responses in the human EEG. Clin Neurophysiol 1999;110:2074-2085.

Herrmann CS, Mecklinger A, Pfeifer E. Gamma responses and ERPs in a visual classification task. Clin Neurophysiol 1999;110:636-642.

Hillyard SA, Anllo-Vento L. Event-related brain potentials in the study of visual selective attention. Proc Natl Acad Sci USA 1998;95:781-787.

Ito TA, Larsen JT, Smith NK, Cacioppo JT. Negative information weighs more heavily on the brain: the negativity bias in evaluative categorizations. J Pers Soc Psychol 1998;75:887-900.

Juergens E, Guettler A, Eckhorn R. Visual stimulation elicits locked and induced gamma oscillations in monkey intracortical- and EEG-potentials, but not in human EEG. Exp Brain Res 1999;129:247-259.

Junghöfer M, Elbert T, Tucker DM, Rockstroh B. Statistical control of artifacts in dense array EEG/MEG studies. Psychophysiology 2000;37:523-532.

Kayser J, Tenke C, Nordby H, Hammerborg D, Hugdahl K, Erdmann G. Event-related potential (ERP) asymmetries to emotional stimuli in a visual half-field paradigm. Psychophysiology 1997;34:414-426.

Keil A, Muller MM, Ray WJ, Gruber T, Elbert T. Human gamma band activity and perception of a gestalt. J Neurosci 1999;19:7152-7161.

Lane RD, Fink GR, Chau PM, Dolan RJ. Neural activation during selective attention to subjective emotional responses. NeuroReport 1997a;8:3969-3972.

Lane RD, Reiman EM, Bradley MM, Lang PJ, Ahern GL, Davidson RJ, Schwartz GE. Neuroanatomical correlates of pleasant and unpleasant emotion. Neuropsychologia 1997b;35:1437-1444.

Lane RD, Reiman EM, Axelrod B, Yun LS, Holmes A, Schwartz GE. Neural correlates of levels of emotional awareness. Evidence of an interaction between emotion and attention in the anterior cingulate cortex. J Cogn Neurosci 1998;10:525-535.

Lang PJ. The motivational organization of emotion: affect-reflex connections. In: Van Goozen SHM, Van de Poll NE, Sergeant JE, editors. Emotions: essays on emotion theory, Hillsdale, NJ: Lawrence Erlbaum, 1994. pp. 61-93.

Lang PJ, Bradley MM, Cuthbert BN. Motivated attention: affect, activation, and action. In: Lang PJ, Simons RF, Balaban MT, editors. Attention and orienting: sensory and motivational processes, Hillsdale, NJ: Lawrence Erlbaum, 1997. pp. 97-135.

Lang PJ, Bradley MM, Cuthbert BN. Emotion, attention, and the startle reflex. Psychol Rev 1990;97:377-395.

Lang PJ, Bradley MM, Cuthbert BN. Emotion, motivation, and anxiety: 
brain mechanisms and psychophysiology. Biol Psychiatry 1998a;44:1248-1263.

Lang PJ, Bradley MM, Fitzsimmons JR, Cuthbert BN, Scott JD, Moulder B, Nangia V. Emotional arousal and activation of the visual cortex: an fMRI analysis. Psychophysiology 1998b;35:199-210.

Luck SJ, Heinze HJ, Mangun GR, Hillyard SA. Visual event-related potentials index focused attention within bilateral stimulus arrays. II. Functional dissociation of P1 and N1 components. Electroenceph clin Neurophysiol 1990;75:528-542.

Mangun GR, Buck LA. Sustained visual-spatial attention produces costs and benefits in response time and evoked neural activity. Neuropsychologia 1998;36:189-200.

Mesulam MM. From sensation to cognition. Brain 1998;121:1013-1052.

Milner PM. A model for visual shape recognition. Psychol Rev 1974;81:521-535.

Mini A, Palomba D, Angrilli A, Bravi S. Emotional information processing and visual evoked brain potentials. Percept Mot Skills 1996;83:143152.

Morris JS, Ohman A, Dolan RJ. A subcortical pathway to the right amygdala mediating 'unseen' fear. Proc Natl Acad Sci USA 1999;96:16801685.

Müller MM, Bosch J, Elbert T, Kreiter A, Sosa MV, Sosa PV, Rockstroh B. Visually induced gamma-band responses in human electroencephalographic activity - a link to animal studies. Exp Brain Res 1996;112:96102.

Müller MM, Junghofer M, Elbert T, Rochstroh B. Visually induced gamma-band responses to coherent and incoherent motion: a replication study. NeuroReport 1997;8:2575-2579.

Müller MM, Keil A, Gruber T, Elbert T. Processing of affective pictures modulates right-hemispheric gamma band EEG activity. Clin Neurophysiol 1999;110:1913-1920.

Murphy ST, Zajonc RB. Affect, cognition, and awareness: affective priming with optimal and suboptimal stimulus exposures. J Pers Soc Psychol 1993;64:723-739.

Palomba D, Angrilli A, Mini A. Visual evoked potentials, heart rate responses and memory to emotional pictorial stimuli. Int J Psychophysiol 1997;27:55-67.

Pfurtscheller G, Stancak Jr A, Neuper C. Event-related synchronization (ERS) in the alpha band - an electrophysiological correlate of cortical idling: a review. Int J Psychophysiol 1996;24:39-46.

Pizzagalli D, Regard M, Lehmann D. Rapid emotional face processing in the human right and left brain hemispheres: an ERP study. NeuroReport 1999;10:2691-2698.

Pulvermüller F. Words in the brain's language. Behav Brain Sci 1999;22:253-336.

Pulvermüller F, Keil A, Elbert T. High-frequency brain activity: perception or active memory? Trends Cogn Sci 1999;3:250-253.
Reiman EM, Lane RD, Ahern GL, Schwartz GE, Davidson RJ, Friston KJ, Yun LS, Chen K. Neuroanatomical correlates of externally and internally generated human emotion. Am J Psychiatry 1997;154:918-925.

Rolls ET. A theory of emotion and consciousness and its application to understanding the neural basis of emotion. In: Gazzaniga MS, editor. The cognitive neurosciences, Cambridge, MA: MIT Press, 1995. pp. 1091-1106.

Royet JP, Zald D, Versace R, Costes N, Lavenne F, Koenig O, Gervais R. Emotional responses to pleasant and unpleasant olfactory, visual, and auditory stimuli: a positron emission tomography study. J Neurosci 2000;20:7752-7759.

Sannita WG. Stimulus-specific oscillatory responses of the brain: a time/ frequency-related coding process. Clin Neurophysiol 2000;111:565583.

Sannita WG, Conforto S, Lopez L, Narici L. Synchronized approximately $15.0-35.0 \mathrm{~Hz}$ oscillatory response to spatially modulated visual patterns in man. Neuroscience 1999;89:619-623.

Schneider F, Gur RE, Mozley LH, Smith RJ, Mozley PD, Censits DM, Alavi A, Gur RC. Mood effects on limbic blood flow correlate with emotional self-rating: a PET study with oxygen-15 labeled water. Psychiatry Res 1995;61:265-283.

Schneider F, Grodd W, Weiss U, Klose U, Mayer KR, Nagele T, Gur RC. Functional MRI reveals left amygdala activation during emotion. Psychiatry Res 1997;76:75-82.

Schupp HT, Cuthbert BN, Bradley MM, Cacioppo JT, Ito T, Lang PJ. Affective picture processing: the late positive potential is modulated by motivational relevance. Psychophysiology 2000;37:257-261.

Shagass C. Electrical activity of the brain. In: Greenbach NS, Sternbach RA, editors. Hanbook of psychophysiology, New York, NY: Holt, Rinehart \& Winston, 1972. pp. 263-328.

Singer W, Engel AK, Kreiter AK, Munk MHJ, Neuenschwander S, Roelfsema PR. Neuronal assemblies: necessity, signature and detectability. Trends Cogn Sci 1997;1:252-261.

Tallon C, Bertrand O, Bouchet P, Pernier J. Gamma-range activity evoked by coherent visual stimuli in humans. Eur J Neurosci 1995;7:12851291.

Tallon-Baudry C, Bertrand O. Oscillatory gamma activity in humans and its role in object representation. Trends Cogn Sci 1999;3:151-162.

Tallon-Baudry C, Bertrand O, Delpuech C, Pernier J. Oscillatory gammaband $(30-70 \mathrm{~Hz})$ activity induced by a visual search task in humans. J Neurosci 1997; 17:722-734.

Tallon-Baudry C, Bertrand O, Peronnet F, Pernier J. Induced gamma-band activity during the delay of a visual short-term memory task in humans. J Neurosci 1998;18:4244-4254.

Tucker DM. Lateral brain function in normal and disordered emotion: interpreting electroencephalographic evidence. Biol Psychol 1984;19:219-235. 